\title{
Importance of processing for physico-chemical and physiological properties of dietary fibre
}

\author{
E. Margareta G.-L. Nyman \\ Applied Nutrition and Food Chemistry, Center for Chemistry and Chemical Engineering, Lund University, PO Box 124, \\ SE-221 00-Lund, Sweden
}

\begin{abstract}
There is considerable loss of DM during wet heat treatment of vegetables, leading to an increase in dietary fibre. Correction for the loss of DM indicates that the effects on total dietary fibre are minor. There is, however, depolymerization of the dietary fibre polysaccharides. The degradation is related to the severity of the heat treatment. Souring, freezing and mild microwave treatment have no effects. The viscosity is in general related to the extent of polymerisation. Microwave treatment has different effects on various cultivars of green beans, and the addition of salt $(\mathrm{NaCl}$ and $\mathrm{CaCl}_{2}$ ) to the boiling water changes the physico-chemical properties of soluble fibre in carrots, depending on the cation. The higher viscosity of the soluble fibre in raw carrots may partly explain the lower glucose and hormonal responses observed in healthy subjects when compared with blanched and microwave-cooked carrots. In studies on rats the amount of butyric acid in the distal colon has been shown to be higher with dietary components containing high amounts of resistant starch. Further, the fermentability is lower and the butyric acid concentration higher with composite foods than with the corresponding purified fibre fractions. In human studies the faecal concentration of butyric acid has been shown to increase in patients with ulcerative colitis when $\beta$-glucan-enriched oat bran (20 g fibre) is added to the diet for 12 weeks. Also, an improvement of symptoms was reported.
\end{abstract}

Dietary fibre: Food processing: Physico-chemical properties: Physiological effects: Butyric acid

A variety of changes take place in the cell-wall polysaccharides during processing and cooking, which affect the physico-chemical properties of dietary fibre and also have nutritional effects. These changes are dependent on several factors. During wet heat treatment of vegetables, for example, the cell membranes are broken down and there is a leakage of DM into the processing water, resulting in an increase in dietary fibre (Nyman et al. 1987b). During such treatment some dietary fibre may also be lost into the boiling water. Many of the alterations that take place during processing may be easily demonstrated by using one of the currently-available fibre analyses. However, in order to better understand the mechanisms behind the physiological effects of dietary fibre there is also a need for more qualitative analyses, such as molecular-weight distribution, viscosity and water-holding capacity. An increased temperature has been reported to break weak bonds between polysaccharide chains (Selvendran \& Robertson, 1994), and split glycosidic linkages in the dietary fibre polysaccharides
(Nyman et al. 1994). As a consequence, the architecture of the fibre matrix may be modified and insoluble fibre solubilized, which in turn can lead to an increased susceptibility to microbial degradation of fibre in the colon (Björck et al. 1984; Nyman et al. 1987a). A higher production of shortchain fatty acids (SCFA) in rats has also been demonstrated in peas after cooking, and the proportion of acetate has been shown to be higher and that of propionate lower (Goodlad \& Mathers, 1992). This finding is interesting, as different SCFA have been shown to be associated with different physiological effects. Propionic acid has been reported to inhibit hepatic cholesterol synthesis from acetic acid and the higher the propionic acid:acetic acid value the more beneficial the effects (Todesco et al. 1991). On the other hand, there is increasing evidence that butyric acid, which is the main energy substrate for the colonic mucosa (Roediger, 1982), protects against the genesis of colonic diseases, such as ulcerative colitis (Cummings, 1997) and also, in the long term, colonic cancer (Scheppach et al. 1995). A reduction in 
the molecular weight of the fibre may also decrease the viscosity and hydration capacity of the fibre, and may possibly lead to changes in metabolic effects (Torsdottir et al. 1989; Tietyen et al. 1990; Gustafsson et al. 1995). Another effect of processing of nutritional impact may be the formation of resistant starch fractions (Björck et al. 1986; Siljeström \& Björck, 1990; Nyman et al. 1991). When designing food products with specific health statements it is important to know more about changes that occur in physico-chemical properties and how these effects can be modulated. The present paper describes studies that provide some of this information. A brief overview is presented of the changes that occur in the physico-chemical properties of dietary fibre, followed by a discussion of some findings that have a nutritional impact (fermentability, SCFA formation, postprandial glucose response).

\section{Results and discussion}

\section{Dietary fibre content}

When vegetables are blanched or cooked they have been shown to lose a considerable amount of DM into the cooking water. On average $25 \%$ is released when carrots (Daucus carota) and Swedes (Brassica napus) are blanched (Nyman et al. 1987b), mainly due to the leakage of lowmolecular-weight carbohydrates into the blanching water (Svanberg et al. 1997a). With subsequent boiling another $20 \%$ is lost. In peas (Pisum sativum), green beans and Brussels sprouts the release is less pronounced (about $10 \%$ ). This leakage of DM generally leads to a considerable increase in the dietary fibre content (Fig. 1 (a)) and, therefore, needs to taken into account, e.g. in databases of
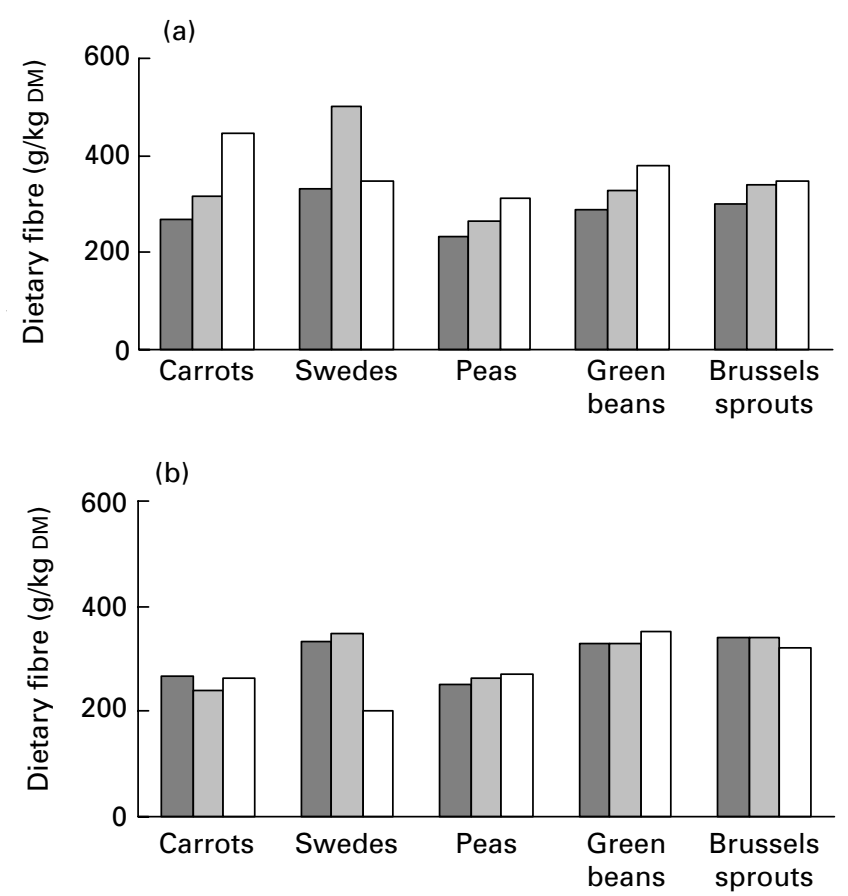

Fig. 1. Dietary fibre contents of raw ( $\equiv$ ), blanched $(\square)$ and boiled $(\square)$ carrots, swedes, peas, green beans and Brussels sprouts before (a) and after (b) correction for losses of DM. (From Nyman et al. 1987b.) dietary fibre content. The fibre per se, however, seems to be relatively unaffected by processing, and correction for the loss of DM indicates only minor effects on total dietary fibre content (Fig 1 (b)). Only when swedes are boiled is there also a leaching of dietary fibre into the boiling water, and on average $40 \%$ of the fibre is lost.

Changes in the total dietary fibre content and the distribution between soluble and insoluble dietary fibre can also be achieved by adding cations to the boiling water (Nyman \& Svanberg, 2002). The presence of $\mathrm{NaCl}$ in the boiling water, at a concentration of $100 \mathrm{mM}$, has been shown to reduce the dietary fibre content of carrots (Fig. 2) as the result of a reduction in mainly uronic acid-containing insoluble polymers. Increasing the concentration of $\mathrm{NaCl}$ $(400 \mathrm{mM})$ has no further effect. Weak bonds between polysaccharide chains are known to be broken during heat treatment (Selvendran \& Robertson, 1994) and $\mathrm{NaCl}$ seems to act as a catalyst in this process. $\mathrm{CaCl}_{2}$ has been shown to have other effects. At low concentration $(100 \mathrm{mM})$ there is a redistribution of soluble dietary fibre to insoluble fibre as a result of cross-linking of pectin molecules. At higher concentrations $(400 \mathrm{mM})$, on the other hand, pectin is degraded, as indicated by the considerable loss of both soluble and insoluble dietary fibre. These effects should be considered when producing foods with a more compact plant structure.

\section{Molecular-weight distribution and viscosity}

Even if total dietary fibre content is relatively unaffected, the functional properties of the fibre may be changed. During heating glycosidic linkages may be broken and the dietary fibre polysaccharides depolymerised. The extent of polymerisation has been shown to be strongly dependent on the extent of the heat treatment, with more extensive degradation resulting from more intense heat treatment (Nyman et al. 1994, Svanberg et al. 1995). Thus, the molecular weight of soluble fibre is highest in raw carrots followed by blanched and then boiled carrots (Svanberg et al. 1995).
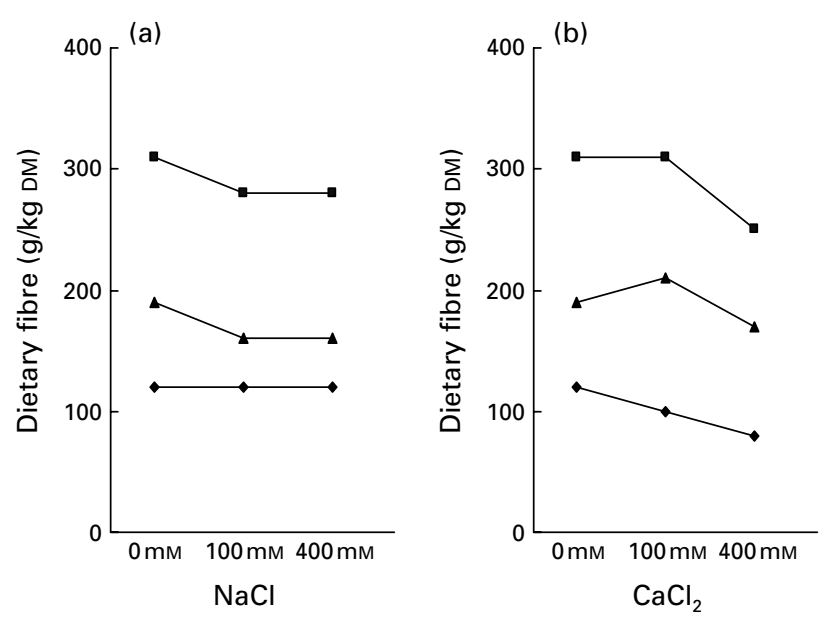

Fig. 2. Effects of cations on the dietary fibre content of boiled carrots. $\mathrm{NaCl}(\mathrm{a})$ or $\mathrm{CaCl}_{2}$ (b) were added to the boiling water at concentrations

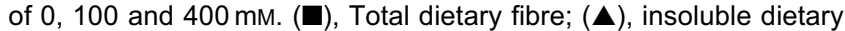
fibre; $(\diamond)$, soluble dietary fibre. (From Nyman \& Svanberg, 2002.) 
However, when blanched carrots are soured or microwave cooked only minor differences in the extent of polymerisation are observed, suggesting that mild microwave treatment as well as souring has minor effects on the molecular weight of soluble fibre in carrots. Similar results have been obtained with raw and frozen material; both exhibiting very similar molecular-weight profiles.

The lower molecular weight of the soluble fibre also results in a lower viscosity. Generally, the maximum viscosity, i.e. the calculated viscosity at the shear rate zero (Morris, 1990), is in accordance with the molecular-weight measurements, and for carrots decreases in the order raw $>$ blanched $>$ boiled. However, in contrast to the effects observed for molecular weight, viscosity is reduced by microwave treatment and souring compared with blanching. It is therefore questionable whether a simple viscosity determination is as appropriate as the molecular-weight determination for ranking changes in physico-chemical properties. A doubling of the molecular weight has been suggested to increase the viscosity about tenfold (Eastwood \& Morris, 1992).

The choice of cultivar may also be important in relation to the molecular-weight distribution and viscosity. The extent of polymerisation following blanching and mild and severe microwave treatment was studied in two batches of green beans (Svanberg et al. 1997b, 1999). The effect of microwave heating differed considerably between the two batches, although the same treatment conditions were used. With one batch the molecular weights for soluble fibre showed a considerable shift towards lower values after mild microwave treatment (Svanberg et al. 1997b), whereas the other batch was essentially unaffected by mild treatment and showed a decrease in molecular weight only after severe heat treatment (Svanberg et al. 1999). The inconsistency in these results may be because the fibre that was most resistant to depolymerization when exposed to heat also had a higher average molecular weight. Thus, for carrot cultivars it has been reported that those containing fibre with a relatively high molecular weight are less affected by heating than those containing fibre with a lower molecular weight (Greve et al. 1994).

In order to produce food products with a high nutritional quality it is important to understand the behaviour of the fibre at the proposed site of action. In the stomach, for example, the low $\mathrm{pH}$ may promote solubilization of the fibre by disruption of $\mathrm{Ca}$ cross-linkages and glycosidic linkages, and in the small intestine the neutral $\mathrm{pH}$ may degrade the pectic polysaccharides by $\beta$-eliminative degradation (Albersheim et al. 1960). A rat experimental model has been used to compare molecular-weight distribution before and after passage through the small and large intestine (Svanberg et al. 1999). To separate effects mediated in the upper part of the intestinal tract from fermentation in the colon, rats were tested with and without an antibiotic drug known to reduce the microbial activity in the hindgut of rats by approximately $90 \%$ (Björck et al. 1986). Faeces were collected from the rats and the molecularweight distribution was analysed. Three main peaks could be distinguished, representing high-, intermediate- and lowmolecular-weight components respectively (Fig. 3). When blanched beans were exposed to microwave heating there

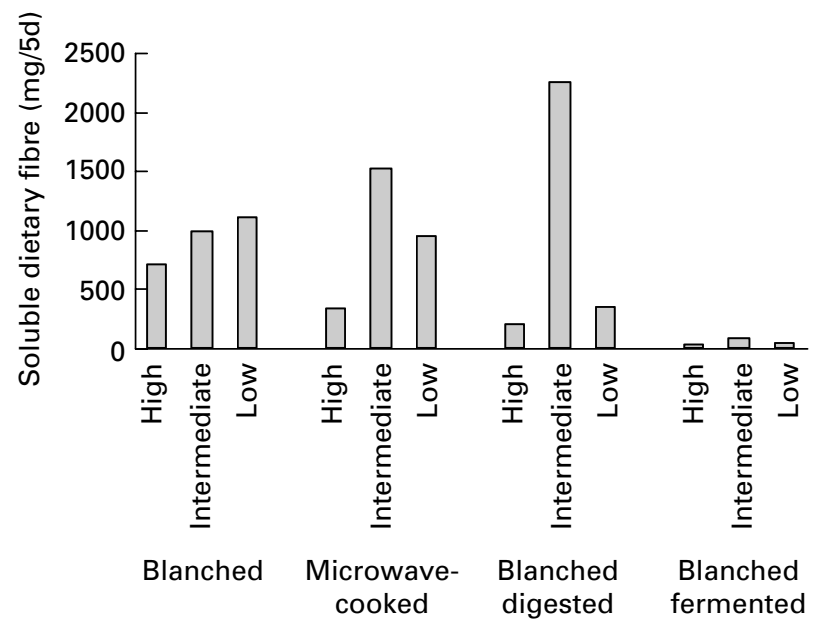

Fig. 3. Molecular-weight distribution of soluble dietary fibre in blanched and microwave-cooked green beans before and after passage through the gastrointestinal tract. High, molecular weight > $700000 \mathrm{Da}$; intermediate, molecular weight $700000-12000 \mathrm{Da}$; low, molecular weight < $12000 \mathrm{Da}$. (From Svanberg et al. 1999.)

was a shift towards intermediate molecular weights and the average weight of the polysaccharides decreased from about 1000000 to 300000 . Depolymerization, but to a greater extent, was also observed when blanched material had passed through the small intestine. Thus, after passage through the small intestine the differences in molecular weight attributable to microwave treatment had already been abolished.

\section{Postprandial glucose response in healthy subjects}

The decreased viscosity of the soluble fibre in carrots following heat treatment may also have nutritional implications in vivo. Postprandial glucose response and hormonal responses have been investigated in healthy subjects after a mixed lunch containing carrots, which were served either raw or following blanching and microwave heating (Gustafsson et al. 1995). The meals, which contained carrots, creamed potatoes, meatballs, lingonberry jam, white bread and light beer, were served in the morning after an overnight fast under standardized conditions to ten healthy volunteers. Both white bread and potatoes are known to produce a high glycaemic index. The amount of dietary fibre derived from the vegetables was $4.4 \mathrm{~g}$, and the energy, digestible carbohydrate, fat and protein contents were similar for the test meals.

Interestingly, raw carrots elicited lower glucose levels and plasma insulin responses than blanched and microwaveheated carrots. It appears, therefore, that ordinary processing and mild cooking of vegetables provided in amounts similar to those normally used may influence the metabolic response to a mixed meal. The lower glucose and hormonal responses with raw carrots may be associated with the higher viscosity of the soluble fibre in these carrots compared with the blanched and microwave-cooked carrots (Svanberg et al. 1995). Another explanation could be that raw carrots have a more compact plant structure and, therefore, are releasing the digestible carbohydrates (mainly sucrose, but also to some 
extent glucose and fructose) more slowly than blanched and microwave-cooked carrots. It should be noted that the meal with the raw carrots enhanced the satiety scores to a greater extent than the blanched and microwave-treated carrots. During blanching and microwave treatment there is a modification of the plant structure; it becomes softer, and less water is bound within the carrots. In this study $126 \mathrm{~g}$ water was bound within blanched and microwave-heated carrots, which was considerably lower that that in raw carrots $(190 \mathrm{~g})$. These differences may lead to disparate influences on the gastric emptying rate and satiety, thus producing another postprandial glucose response. It is possible, therefore, that viscosity, and hydration capacity, which is also associated with insoluble fibre, is important in the metabolic response to the fibre. Thus, modification of the plant structure, similar to that achieved with low concentrations of $\mathrm{CaCl}_{2}$ (see p. 188), may be of importance.

\section{Fermentation and short-chain fatty acid formation in the rat hindgut}

Various types of dietary fibre and resistant starch fractions have been shown to produce different SCFA profiles when fermented in the colon. However, SCFA patterns have been studied mainly using single substrates. Studies in which the impact of the nature of the substrate and the effects of processing have been investigated are scarce, although more relevant to the human diet.

To obtain test samples differing in the distribution and content of indigestible carbohydrates as well as in botanical structure the same batch of red kidney beans (Phaseolus vulgaris) was processed in different ways (Henningsson et al. 2001). Hindgut fermentability and production of SCFA were then studied using the rat model that has been used previously to study fermentability (Nyman \& Asp, 1982). Bean flours with low or high contents of resistant starch, mainly raw and physically-indigestible starch, were obtained by milling the beans before or after boiling. The oligosaccharide content was modulated by freeze-drying both with and without the water used during autoclaving. Six diets (two with higher levels of indigestible carbohydrates) were prepared from the flours.

The total fermentability of the indigestible carbohydrates was shown to be high and similar with all diets (80-87\%). Furthermore, the fermentability was higher for autoclaved beans than for boiled products $(P<0 \cdot 05)$. The relative proportions of acetic, propionic and butyric acids in the caecum were similar for all diets, but with a comparatively high proportion of butyric acid compared with that obtained with single substrates tested in the same rat model (Berggren et al. 1993). A possible explanation for this finding is that beans contain high amounts of raffinose, or that a mixture of indigestible carbohydrates was used. Pure raffinose has previously been shown to produce large amounts of caecal butyrate (Berggren et al. 1993), and combinations of substrates have also been reported to increase the amount of butyric acid formed (Topping et al. 1985). Although there were minor differences between the substrates in SCFA formation in the caecum, there were marked differences in the distal colon. The diet containing intact cells and the highest amount of resistant starch gave the highest

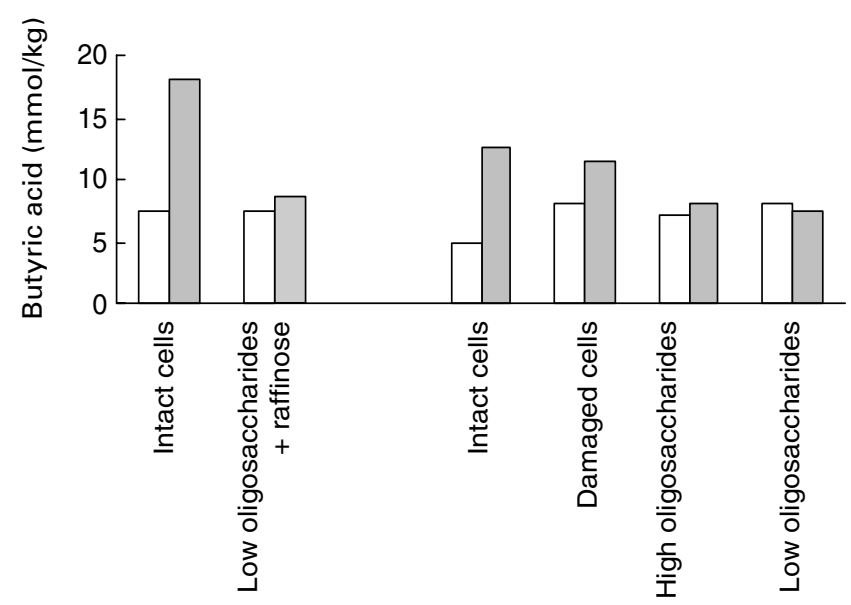

Fig. 4. Butyric acid concentration in the proximal $(\square)$ and distal (四) colon of rats fed diets prepared from flours obtained from a batch of red kidney beans subjected to different methods of processing. For details, see p. 190. (From Henningsson et al. 2001.)

percentage of butyric acid (24 v. 12-17 with the other test diets). Also, the two diets containing high amounts of resistant starch promoted a higher butyric acid concentration in the distal colon than in the proximal colon, whereas it remained constant or decreased in the case of the other diets (Fig. 4). This outcome must be considered to be positive, as most colonic diseases occur in the distal part of the colon.

In another study the fermentability and the production of SCFA from dietary fibre in composite food products in normal use (potato powder, apples and oat flakes) were compared with commercial fibre fractions from similar sources (potato fibre, apple pectin and oat fibre) (Henningsson et al. 2002). The composition of the indigestible carbohydrates was similar for the two oat products and the two potato products. However, potato powder contained a much higher level of resistant starch than the potato fibre ( 220 and $70 \mathrm{~g} / \mathrm{kg}$ respectively). The composition of the fibre in apples was also much more complex than the pure pectin, and in addition to uronic acids the fibre also contained large amounts of polymers consisting of xylose, galactose and glucose. The fermentability of the indigestible carbohydrates was high for all diets (79-90\%), but total fermentability tended to be higher with the commercial fibre fractions than with the composite food items. This difference was significant for the apple pectin $(P<0 \cdot 05)$ and the oat fibre $(P<0 \cdot 01)$ but not for the potato fibre.

All substrates except the pure apple pectin produced intermediate to high amounts of butyric acid in the caecum (\% SCFA; 15-22 v. 8 for pectin). In the distal colon, however, it was shown that potato powder, i.e. the diet with the highest amount of resistant starch, also produced the highest amounts of butyric acid (\% SCFA; 19 v. 8-14). Furthermore, the composite foods, i.e. potato powder, apples and oat flakes, promoted higher proportions of butyric acid in the distal colon as compared with the purified fibre fractions $(P<0 \cdot 05)$. For apples, the higher proportion of butyric acid may be because apples contain considerable amounts of sorbitol and fructose. Sorbitol has been reported to yield high proportions of butyric acid during in vitro fermentation (Mortensen et al. 1988) and fructose- 
containing oligosaccharides have been shown to produce high proportions of butyric acid in the caecum of rats (Levrat et al. 1991; Berggren et al. 1993; Roland et al. 1995). At high intakes of fructose this sugar may also reach the colon for fermentation, and a high proportion of butyric acid in the caecum with apples in the study (Henningsson et al. 2002) may be the result of the fermentation of fructose. The high proportion of butyric acid in the distal colon with potato powder may be explained by its high content of resistant starch (Cummings et al. 1996; Martin et al. 1998). Resistant starch has been shown to produce high proportions of butyric acid during, for example, in vitro fermentations (Englyst et al. 1987). The two oat products, however, had a very similar dietary fibre composition, but oat flakes produced a higher proportion of butyric acid. There was a similar outcome for potato powder and apples, compared with potato fibre and pectin. The architecture of the fibre matrix is more complex in composite foods than in pure fibre fractions, and it is possible that this factor explains the slower fermentability and higher production of butyric acid in the distal colon.

\section{Studies on patients with ulcerative colitis}

Subjects with ulcerative colitis have an impaired capacity to oxidise butyric acid, and when enemas containing butyrate are distributed to these patients the damage score for the colonic mucosa has been shown to fall dramatically (Scheppach et al. 1992). Surprisingly, there are no specific dietary recommendations for individuals with ulcerative colitis, but where dietary advice is given these patients are usually prescribed a diet containing low amounts of dietary fibre. However, as butyric acid can be obtained from the fermentation of some types of dietary fibre, it was decided to investigate the efficacy of dietary regulation of colonic butyrate production. Previous studies on rats had shown that pure $\beta$-glucans yielded high proportions of butyric acid in the rat hindgut (Berggren et al. 1993). On this basis a pilot study of patients with ulcerative colitis was undertaken to investigate whether faecal butyrate could be increased by adding a $\beta$-glucan-enriched oat bran to the diet (Hallert et al. 2003).

The twenty-three patients who participated in the study had been in clinical and endoscopic remission for at least 3 months. The intervention lasted for 12 weeks and the patients received four slices of oat bread every day and some oat bran served in water or yoghurt. The supplement represented a daily intake of $20 \mathrm{~g}$ dietary fibre, which is similar to the average intake of adults in most Western countries.

The greatest impact of the oat-bran intervention was on butyric acid concentration. Faecal butyrate had increased after 4 weeks in fifteen of the nineteen patients that completed the study, and over the 12-week period in thirteen patients. Interestingly, only the concentration of butyric acid was changed by oat-bran supplementation; the other SCFA were unaffected. Furthermore, patients reporting abdominal pain or reflux at the beginning of the study showed marked improvement at 12 weeks. Thus, for patients with ulcerative colitis there is some evidence that butyric acid formation can be increased by diet. Furthermore, a diet rich in dietary fibre is apparently not harmful for these patients, as there were no reports of clinical impairment.

In conclusion, processing can have a marked effect on dietary fibre, but by selecting a suitable cultivar and appropriate processing conditions it may be possible to achieve specific health effects. However, more data are needed before any clear-cut conclusions can be drawn.

\section{Acknowledgements}

This work was conducted predominantly at Lund University, Sweden and supported by grants from Direktör Albert Påhlsson's foundation, LiFT-programme, Nestec Ltd Switzerland, Swedish Foundation of Agricultural Research and VL-foundation. The author is indebted to her collaborators, particularly Professor I. Björck, Associate Professor C. Hallert, Dr M. Svanberg and Dr Å. Henningsson.

\section{References}

Albersheim P, Neukom H \& Deuel H (1960) Splitting of pectin chain molecules in neutral solutions. Archives of Biochemistry and Biophysics 90, 46-51.

Berggren AM, Björck IME \& Nyman EMG-L (1993) Short-chain fatty acid content and $\mathrm{pH}$ in caecum of rats given various sources of carbohydrates. Journal of the Science of Food and Agriculture 63, 397-406.

Björck I, Nyman M \& Asp N-G (1984) Extrusion cooking and dietary fibre: effects on dietary fibre content and on degradation in the rat intestinal tract. Cereal Chemistry 61, 174-179.

Björck IM, Nyman M, Pedersen B, Siljeström M, Asp N-G \& Eggum BO (1986) On the digestibility of starch in wheat bread Studies in vitro and in vivo. Journal of Cereal Science 4, 1-11.

Cummings JH (1997) Short-chain fatty acid enemas in the treatment of distal ulcerative colitis. European Journal of Gastroenterology and Hepatology 9, 149-153.

Cummings JH, Beatty ER, Kingman SM, Bingham SA \& Englyst HN (1996) Digestion and physiological properties of resistant starch in the human large bowel. British Journal of Nutrition 75, 733-747.

Eastwood MA \& Morris ER (1992) Physical properties of dietary fiber that influence physiological function: a model for polymers along the gastrointestinal tract. American Journal of Clinical Nutrition 55, 436-442.

Englyst HN, Hay S \& Macfarlane GT (1987) Polysaccharide breakdown by mixed populations of human faecal bacteria. FEMS Microbial Ecology 95, 163-171.

Goodlad JS \& Mathers JC (1992) Digestion of complex carbohydrates and large bowel fermentation in rats fed on raw and cooked peas (Pisum sativum). British Journal of Nutrition 67, $475-488$.

Greve LC, McArdle RN, Gohlke JR \& Labavitch JM (1994) Impact of heating on carrot firmness: changes in cell wall components. Journal of Agricultural and Food Chemistry 42, 2900-2906.

Gustafsson K, Asp N-G, Hagander B, Nyman M \& Schweizer T (1995) Influence of processing and cooking of carrots in mixed meals on satiety, glucose and hormonal response. International Journal of Food Science and Nutrition 46, 3-12.

Hallert C, Björck I, Nyman M, Pousette A, Grännö C \& Svensson $H$ (2003) Increasing the fecal butyrate in ulcerative colitis patients by diet. A pilot study. Inflammatory Bowel Diseases 9 (In the Press).

Henningsson ÅM, Nyman EMG-L \& Björck IME (2001) Content of short-chain fatty acids in the hindgut of rats fed processed 
bean (Phaseolus vulgaris) flours varying in distribution and content of indigestible carbohydrates. British Journal of Nutrition 86, 379-389.

Henningsson ÅM, Nyman EMG-L \& Björck IME (2002) Shortchain fatty acid content in the hindgut of rats fed various composite foods and commercial dietary fibre fractions from similar sources. Journal of the Science of Food and Agriculture 82, 385-393.

Levrat MA, Remesy C \& Demigne C (1991) High propionic acid fermentations and mineral accumulation in the cecum of rats adapted to different levels of inulin. Journal of Nutrition 121, $1730-1737$

Martin LJM, Dumon HJW \& Champ MMJ (1998) Production of short-chain fatty acids from resistant starch in a pig model. Journal of the Science of Food and Agriculture 77, 71-80.

Morris ER (1990) Shear-thinning of 'random coil' polysaccharides: characterisation by two parameters from a simple linear plot. Carbohydrate Polymers 13, 85-96.

Mortensen PB, Holtug K \& Rasmussen HS (1988) Short-chain fatty acid production from mono- and disaccharides in a fecal incubation system: implications for colonic fermentation of dietary fiber in humans. Journal of Nutrition 118, 321-325.

Nyman EMG-L \& Svanberg SJM (2002) Modification of physicochemical properties of dietary fibre in carrots by mono- and divalent cations. Food Chemistry 76, 273-280.

Nyman EMG-L, Svanberg SJM \& Asp N-G (1994) Molecular weight distribution and viscosity of water-soluble dietary fibre isolated from green beans, Brussels sprouts and green peas following different types of processing. Journal of the Science of Food and Agriculture 66, 83-91.

Nyman M \& Asp N-G (1982) Fermentation of dietary fibre components in the rat intestinal tract. British Journal of Nutrition 47, 357-366.

Nyman M, Björck I, Håkansson B \& Asp N-G (1987a) Popping of whole-grain wheat: effect on dietary fibre degradation in the rat intestinal tract. Journal of Cereal Science 5, 67-72.

Nyman M, Pålsson K-E \& Asp N-G (1987b) Effects of processing on dietary fibre in vegetables. Lebensmittel Wissenschaft und Technologie 20, 29-36.

Nyman M, Schweizer TF, Pålsson KE \& Asp NG (1991) Effects of processing on fermentation of dietary fibre in vegetables by rats. Lebensmittel Wissenschaft und Technologie 24, 433-441.

Roediger WE (1982) Utilization of nutrients by isolated epithelial cells of the rat colon. Gastroenterology 83, 424-429.

Roland N, Nugon-Baudon L, Andrieux C \& Szylit O (1995) Comparative study of the fermentative characteristics of inulin and different types of fibre in rats inoculated with a human whole faecal flora. British Journal of Nutrition 74, 239-249.

Scheppach W, Bartram HP \& Richter F (1995) Role of short-chain fatty acids in the prevention of colorectal cancer. European Journal of Cancer 31 A, 1077-1080.

Scheppach W, Sommer H, Kirchner T, Paganelli GM, Bartram P, Christl S, Richter F, Dusel G \& Kasper H (1992) Effect of butyrate enemas on the colonic mucosa in distal ulcerative colitis. Gastroenterology 103, 51-56.

Selvendran RR \& Robertson JA (1994) Dietary fibre in foods: amount and type. In Physico-chemical Properties of Dietary Fibre and Effect of Processing on Micronutrients Availability, pp. 11-19 [R Amadò, JL Barry and W Frølich, editors]. Luxembourg: COST 92 Directorate-general XIII.

Siljeström M \& Björck I (1990) Digestible and undigestible carbohydrates in autoclaved legumes, potatoes and corn. Food Chemistry 38, 145-152.

Svanberg SJM, Gustafsson KBH, Suortti T \& Nyman EMG-L (1995) Molecular weight distribution, measured by HPSEC, and viscosity of water-soluble dietary fiber in carrots following different types of processing. Journal of Agricultural and Food Chemistry 43, 2692-2697.

Svanberg SJM, Nyman EMG-L, Nilsson R \& Nilsson T (1997a) Effects of boiling and storage on dietary fibre and digestible carbohydrates in various cultivars of carrots. Journal of the Science of Food and Agriculture 73, 245-254.

Svanberg SJM, Suortti T \& Nyman EMG-L (1997b) Physicochemical changes in dietary fiber of green beans after repeated microwave treatments. Journal of Food Science 62, 1006-1010.

Svanberg SJM, Suortti T \& Nyman EMG-L (1999) Intestinal degradation of dietary fibre in green beans - effects of microwave treatments. International Journal of Food Science and Nutrition 50, 245-253.

Tietyen JL, Nevins DJ \& Schneeman BO (1990) Characterization of the hypocholesterolic potential of oat bran. FASEB Journal 4, A527.

Todesco T, Rao AV, Bosello O \& Jenkins DJ (1991) Propionate lowers blood glucose and alters lipid metabolism in healthy subjects. American Journal of Clinical Nutrition 54, 860-865.

Topping DL, Illman RJ \& Trimble RP (1985) Volatile fatty acids concentrations in rats fed diets containing gum arabic and cellulose separately and as a mixture. Nutrition Reports International 32, 809-814.

Torsdottir I, Alpsten M, Andersson H \& Einarsson S (1989) Dietary guar gum effects on postprandial blood glucose, insulin and hydroxyproline in humans. Journal of Nutrition 119, 1925-1931. 